\title{
Modeling the 802.11 Distributed Coordination Function in Non-Saturated Conditions
}

\author{
Ken Duffy, David Malone, and Douglas J. Leith
}

\begin{abstract}
Analysis of the 802.11 CSMA/CA mechanism has received considerable attention recently. Bianchi [1] presents an analytic model under a saturated traffic assumption. Bianchi's model is accurate, but typical network conditions are nonsaturated. We present an extension of his model to a nonsaturated environment. Its predictions are validated against simulation and are found to accurately capture many interesting features of non-saturated operation.
\end{abstract}

Index Terms-Wireless LAN, IEEE 802.11 MAC, nonsaturated traffic, performance evaluation.

\section{INTRODUCTION}

$\mathbf{T}$ HE 802.11 wireless LAN standard has been widely deployed during recent years and has received considerable research attention. The 802.11 MAC layer uses a CSMA/CA algorithm with binary exponential back-off to regulate access to the shared wireless channel. While this algorithm has been the subject of numerous empirical studies, an analytic framework for reasoning about its properties remains notably lacking. Developing analysis tools is desirable not only because of the wide deployment of 802.11 equipment but also because the CSMA/CA mechanism continues to play a key role in new standards proposals such as 802.11e. A key difficulty in the mathematical modeling the 802.11 MAC lies in the very large number of states that may exist (scaling exponentially with the number of nodes). In his seminal paper, Bianchi [1] addressed this difficulty by assuming that (i) every node is saturated (i.e. always has a packet to be transmitted) and (ii) the packet collision probability is constant regardless of the state or station considered. Provided that every node is indeed saturated, the resulting model is remarkably accurate. Unfortunately, the saturation assumption is unlikely to be valid in most real 802.11 networks. Data traffic such as web and email is typically bursty in nature while streaming traffic such as voice operates at relatively low rates and often in an on-off manner. Hence, for most real traffic the demanded transmission rate is variable with significant idle periods. Our aim is to derive a mathematical model that relaxes the restriction to saturated operation while retaining as much as possible of the attractive simplicity of Bianchi's model (in particular, the ability to obtain analytic relationships).

\section{ANALYSIS}

Bianchi [1] presents a Markov model where each station is modeled by a pair of integers $(i, k)$. The back-off stage,

Manuscript received January 13, 2005. The associate editor coordinating the review of this letter and approving it for publication was Dr. Nikos Nikolaou. This work was supported by Science Foundation Ireland grant 03/IN3/I396.

The authors are with the Hamilton Institute, National University of Ireland, Maynooth, Co. Kildare, Ireland (e-mail: David.Malone@nuim.ie).

Digital Object Identifier 10.1109/LCOMM.2005.08019. $i$, starts at 0 at the first attempt to transmit a packet and is increased by 1 every time a transmission attempt results in a collision, up to a maximum value $m$. It is reset after a successful transmission. The counter, $k$ is initially chosen uniformly between $\left[0, W_{i}-1\right]$, where $W_{i}=2^{i} W_{0}$ is the range of the counter. While the medium is idle, the counter is decremented. Transmission is attempted when $k=0$.

We assume each station can buffer one packet and there is a constant probability $q$ of at least one packet arriving per state. Thus we introduce states $(0, k)_{e}$ for $k \in\left[0, W_{0}-1\right]$, representing a node which has transmitted a packet, but has none waiting. Note that $i=0$ in all such states, because if $i>0$ then a collision has occurred, so we must have a packet awaiting transmission.

We now derive relationships between: $p$, the probability of collision; $P$, the Markov chain's transition matrix; $b$, the stationary distribution; and $\tau$, the transmission probability per station. These relationships can be solved for $p$ and $\tau$, and network throughput predicted. It is important to note that the Markov chain's evolution is not real-time, and so the estimation of throughput requires an estimate of the average state duration.

The simplest transitions are those where the counter is nonzero. If we have a packet, then the only possible change is that the counter decrements. If we do not have a packet, the counter will decrement, but a packet may also arrive with probability $q$. Thus, for $0<k<W_{i}$ we have

$$
\begin{aligned}
0<i \leq m, \quad P[(i, k-1) \mid(i, k)] & =1, \\
P\left[(0, k-1)_{e} \mid(0, k)_{e}\right] & =1-q, \\
P\left[(0, k-1) \mid(0, k)_{e}\right] & =q .
\end{aligned}
$$

If the counter reaches 0 and a packet has arrived, we begin a transmission. We assume there is a probability $p$ that another node transmits at the same time, resulting in a collision and an increase in the back-off stage. Thus for $0 \leq i \leq m$ and $k \geq 0$ we have

$$
\begin{aligned}
P\left[(0, k)_{e} \mid(i, 0)\right] & =\frac{(1-p)(1-q)}{W_{0}}, \\
P[(0, k) \mid(i, 0)] & =\frac{(1-p) q}{W_{0}}, \\
P[(\min (i+1, m), k) \mid(i, 0)] & =\frac{{ }^{2}}{W_{\min (i+1, m)}} .
\end{aligned}
$$

The most complex transitions are from the $(0,0)_{e}$ state, where the countdown is complete, but we have no packet to send. If no packet arrives, we stay in this state. If a packet arrives, the new state depends on the current state of the medium: if the medium is idle we may begin transmission, which may result in a successful transmission or a collision; if the medium is busy, the 802.11 MAC begins another stage-0 back-off. This 
TABLE I

PARAMETERS VALUES FOR MODEL AND SIMULATION.

\begin{tabular}{|lr|cr|ll|}
\hline$W_{0}$ & 31 & $E$ & $407 \mathrm{us}$ & $T_{s}$ & $986 \mathrm{us}$ \\
$m$ & 5 & $\sigma$ & $20 \mathrm{us}$ & $T_{c}$ & $986 \mathrm{us}$ \\
\hline
\end{tabular}

gives

$$
\begin{aligned}
P\left[(0,0)_{e} \mid(0,0)_{e}\right] & =1-q+\frac{q(1-\tau)^{n-1}(1-p)}{W_{0}}, \\
k>0, \quad P\left[(0, k)_{e} \mid(0,0)_{e}\right] & =\frac{q(1-\tau)^{n-1}(1-p)}{W_{0}}, \\
k \geq 0, \quad P\left[(1, k) \mid(0,0)_{e}\right] & =\frac{q(1-\tau)^{n-1} p}{W_{1}}, \\
k \geq 0, \quad P\left[(0, k) \mid(0,0)_{e}\right] & =\frac{q\left(1-(1-\tau)^{n-1}\right)}{W_{0}} .
\end{aligned}
$$

Note that we have used $(1-\tau)^{n-1}$ as the probability that the medium is idle. As noted by Bianchi, $1-p=(1-\tau)^{n-1}$, thus our transition probabilities only depend on $p$ and $q$.

Solving for the stationary distribution, $b$, yields (after lengthy algebra)

$$
\begin{aligned}
& 1 / b_{(0,0)_{e}}=(1-q)+\frac{q^{2} W_{0}\left(W_{0}+1\right)}{2\left(1-(1-q)^{W_{0}}\right)} \\
& +\frac{q\left(W_{0}+1\right)}{2(1-q)}\left(\frac{q^{2} W_{0}}{1-(1-q)^{W_{0}}}+p(1-q)-q(1-p)^{2}\right) \\
& +\frac{p q^{2}}{2(1-q)(1-p)}\left(\frac{W_{0}}{1-(1-q)^{W_{0}}}-(1-p)^{2}\right) \\
& \left(2 W_{0} \frac{1-p-p(2 p)^{m-1}}{1-2 p}+1\right)
\end{aligned}
$$

and

$$
\begin{aligned}
\tau & =\sum_{i=0}^{m} b_{(i, 0)}+b_{(0,0)_{e}} q(1-p) \\
& =b_{(0,0)_{e}} \frac{q^{2}}{1-q}\left(\frac{W_{0}}{(1-p)\left(1-(1-q)^{W_{0}}\right)}-(1-p)\right) .
\end{aligned}
$$

For given values of $q, W_{0}, n$ and $m$ we may solve (2) against $1-p=(1-\tau)^{n-1}$ to determine $p$ and $\tau$. In the limit $q \rightarrow 1$, our model yields the same value for $\tau$ and $p$ as Bianchi's saturated model.

The expression for throughput is the same as in [1],

$$
S=\frac{P_{s} P_{t r} E}{\left(1-P_{t r}\right) \sigma+P_{t r} P_{s} T_{s}+P_{t r}\left(1-P_{s}\right) T_{c}},
$$

where $P_{t r}=1-(1-\tau)^{n}, P_{s}=n \tau(1-\tau)^{n-1} / P_{t r}, E$ is the time spent transmitting payload data, $\sigma$ is the time for the counter to decrement, $T_{s}$ is the time for a successful transmission and $T_{c}$ is the time for a collision. The denominator of this fraction is the expected duration of a state in the Markov chain in real-time, which we denote $T$.

To match the model with experiment we must relate $q$ to offered packet load. Modeling a saturated system, i.e. there is always a packet awaiting service, is achieved by setting $q=1$. If packets arrive in a Poisson manner with exponentially distributed inter-packet arrival times with rate $\lambda$, then $1-q$ is the probability no packet arrives in a typical slot of length $T$. That is $1-q=\exp (-\lambda T)$ and therefore $q=1-\exp (-\lambda T)$.

\section{VALIDATION}

The model was verified against TU-Berlin's [2] ns2 802.11 simulator. MAC parameter values (corresponding to 802.11b) and packet sizes used are in Table I. Varying numbers of stations, with a small buffer, were simulated. In the first set of simulations, arrivals to each station are Poisson.

Figs. 1 and 2 show predicted and simulated throughput against offered load, for a different numbers of wireless nodes

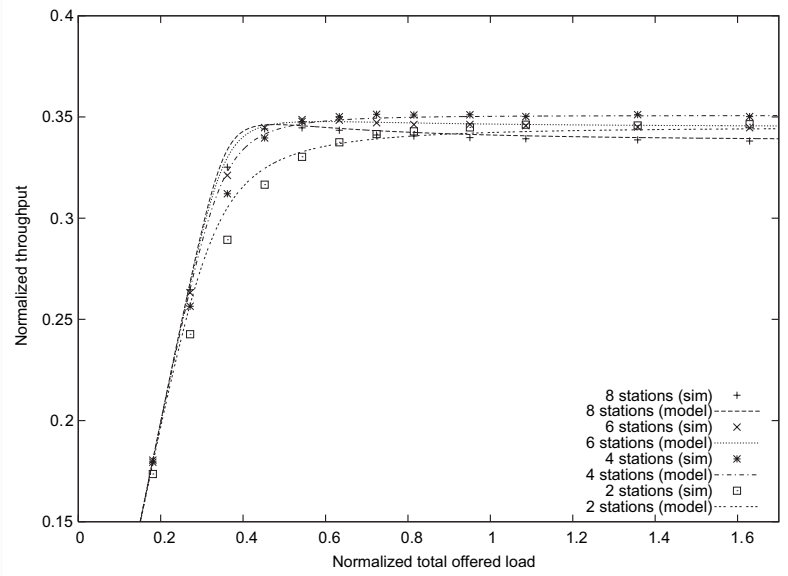

Fig. 1. Throughput vs. offered load for small numbers of nodes. For rates below those shown, there is agreement.

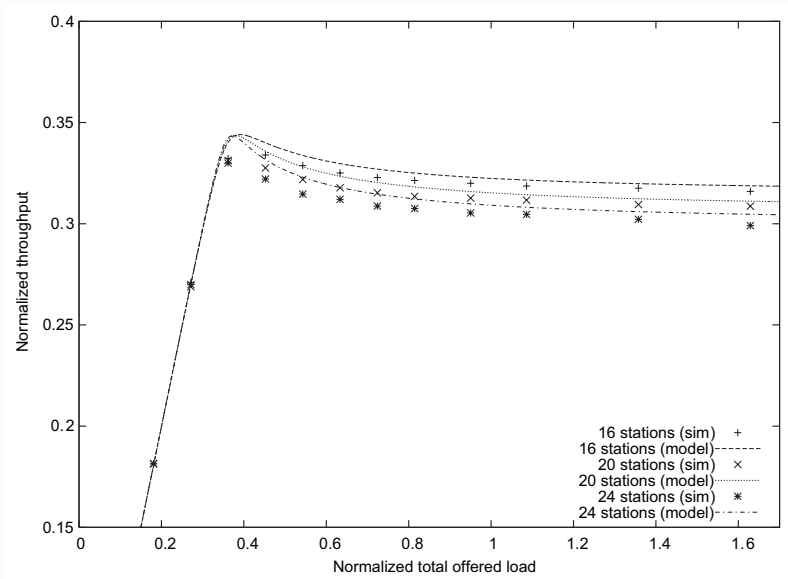

Fig. 2. Throughput vs. offered load for larger numbers of nodes. For rates below those shown, there is agreement.

(arrival rates are normalized by the data rate of $11 \mathrm{Mbs}$ ). Collision probabilities corresponding to Fig. 1 are shown in Fig. 3 (similar accuracy is obtained for the conditions used in Fig. 2). The model accurately captures important features. In particular,

- the linear relationship (with slope 1) between throughput and offered load under low loads.

- the limiting behavior of throughput at high offered loads (corresponding to saturation).

- the complex transition between under-loaded and saturated regimes is accurately captured. For small numbers of nodes, saturation throughput is the peak throughput. For larger numbers of nodes, the throughput falls as we approach saturation and peak throughput is achieved before saturation. The offered load at which this peak occurs is relatively insensitive to the number of nodes.

In the foregoing plots, packets arrivals are Poisson, yielding independent arrivals at a specified mean rate. However, we have found that similar results hold for a range of traffic types. To illustrate this, we briefly present results for simulated voice traffic with silence suppression. Following [3], we generate a $64 \mathrm{kbs}$ on-off traffic stream with on and off periods expo- 


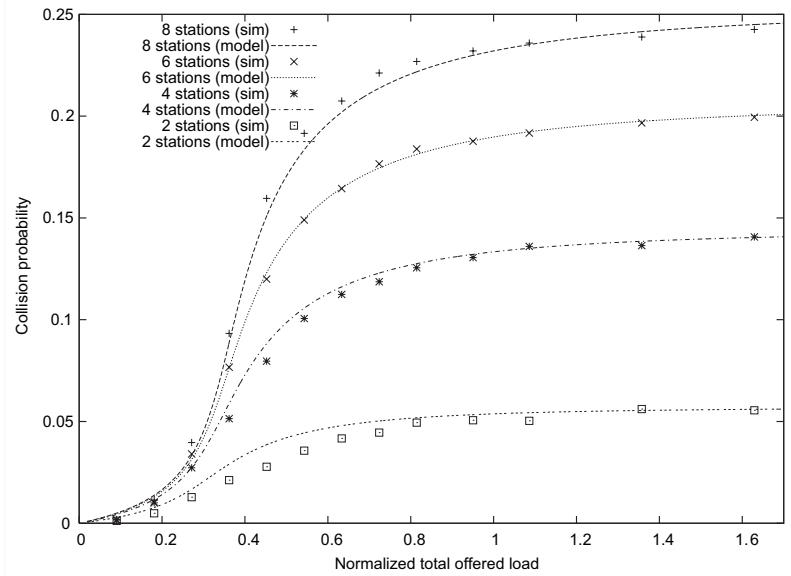

Fig. 3. Collision probability vs. offered load for small numbers of nodes.

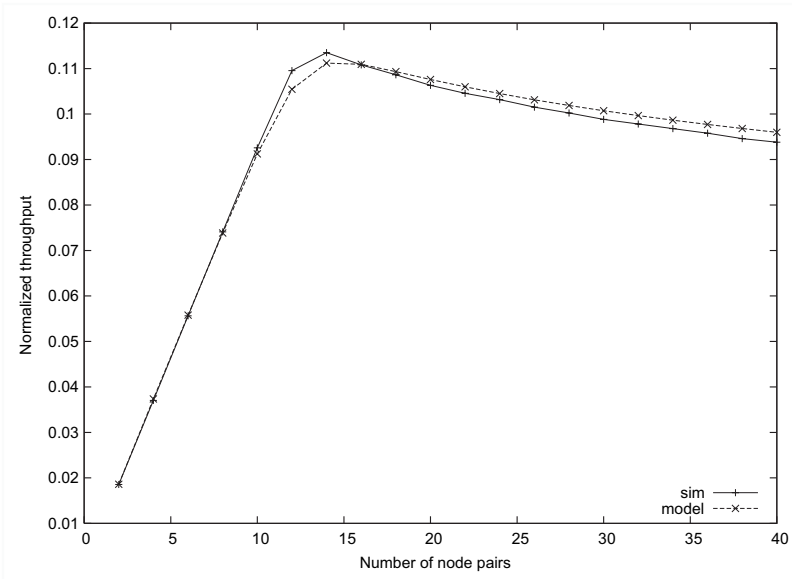

Fig. 4. Throughput vs. numbers of node-pairs sending 64kbps on-off traffic streams.

nentially distributed with mean $1.5 \mathrm{~s}$, subject to a minimum of $240 \mathrm{~ms}$. Traffic is between pairs of nodes to account for the two-way correlated nature of voice conversations; the on/off periods of one node correspond to the off/on periods of another. We apply our model to node-pairs when making predictions. Predicted and simulated throughput versus the number of node-pairs are shown in Fig. 4, where it can be seen that our model is remarkably accurate.

\section{CONSIDERATIONS}

It is easy to consider small variations on this model, such as disallowing packet arrival immediately after transmission, ignoring carrier sense in state $(0,0)_{e}$, or by limiting the number of retransmission attempts. We have investigated these possibilities and found that they result in numerical changes that are not significant.

Two important assumptions of the model are constant probability of arrival per state and small interface buffers. The accuracy of the model predictions for a range of traffic types, as noted previously, suggests there is a useful robustness with respect to the first assumption. We have found that the predictions are more sensitive to the presence of large buffers. It is possible to introduce extra states to model longer queues, and also to allow variable packet arrival probabilities per state. Owing to space restrictions this is beyond the scope of this paper.

\section{RELATED WORK}

There are alternative approaches to non-saturated modeling. In [4] a modification of [1] is considered where a probability of not transmitting is introduced that represents a station having no data to send. The model is not predictive as this probability is not known as a function of load and must be estimated from simulation. In [5] idle states are added after packet transmission to represent bursty arrivals in a way that does not account for postbackoff. In [6] a model where states are of fixed real-time length is introduced, but does not capture the feature of a pre-saturation throughput peak. In [7] a model incorporating postbackoff is presented, but not solved explicitly. In [8] a non-Markov model is developed, but is based on an unjustified assumption that the saturated setting provides good approximation to certain unsaturated quantities.

\section{CONCLUSION}

We present a model of the 802.11 MAC layer in nonsaturated conditions. It is analytically tractable yet remarkably powerful. It is shown to be in quantitative and qualitative agreement with detailed simulations, yielding accurate predictions of throughput and collision probability. It captures important features of non-saturated operation (e.g. throughput may be higher in non-saturated operation than saturated). It is accurate for a range of traffic types. This is illustrated with voice calls (to the authors' knowledge this is the first demonstration of an analytic model of voice calls in 802.11). The model is interesting not only because of wide deployment of 802.11 equipment and prevalence of non-saturated operation in wireless networks, but also because the CSMA/CA mechanism plays a central role in new standards such as 802.11e [9].

\section{REFERENCES}

[1] G. Bianchi, "Performance analysis of IEEE 802.11 distributed coordination function," IEEE J. Select. Areas Commun., vol. 18, pp. 535-547, Mar. 2000.

[2] S. Wiethölter and C. Hoene, "Design and verification of an IEEE 802.11e EDCF simulation model in ns-2.26," Technische Universität Berlin, Tech. Rep. TKN-03-019, Nov. 2003.

[3] A. Markopoulou, F. Tobagi, and M. Karam, "Assessing the quality of voice communications over Internet backbones," IEEE Trans. Networking, vol. 11, pp. 747-760, Oct. 2003.

[4] G.-S. Ahn, A. T. Campbell, A. Veres, and L.-H. Sun, "Supporting service differentiation for real-time and best-effort traffic in stateless wireless ad hoc networks (SWAN)," IEEE Trans. Mobile Computing, vol. 1, pp. 192207, 2002.

[5] M. Ergen and P. Varaiya, "Throughput analysis and admission control in IEEE 802.11a," Mobile Networks and Applications, MONET Special Issue on WLAN Optimization at the MAC and Network Levels, to appear.

[6] A. Zaki and M. El-Hadidi, "Throughput analysis of IEEE 802.11 DCF under finite load traffic," in Proc. First International Symposium on Control, Communications and Signal Processing, 2004, pp. 535-538.

[7] G. Cantieni, Q. Ni, C. Barakat, and T. Turletti. "Performance analysis under finite load and improvements for multirate 802.11b," Elsevier Computer Commun. Journal, vol. 28, pp. 1095-1109, June 2005.

[8] O. Tickoo and B. Sikdar, "A queueing model for finite load IEEE 802.11 random access," in Proc. IEEE International Conference on Communications, vol. 1, June 2004, pp. 175-179.

[9] Wirless LAN Medium Access Control (MAC) and Physical Layer (PHY) Specifications: Medium Access Control (MAC) enhancements for Quality of Service $(Q o S)$, IEEE std 802.11e-draft ed., IEEE. 